\title{
Increases of Corporal Temperature as a Risk Factor of Atherosclerotic Plaque Instability
}

\author{
GUSTAVO V. GUINEA, JOSE M. ATIENZA, PANAYOTIS FANTIDIS, FRANCISCO J. ROJO, ARANZAZU ORTEGA, \\ MONICA TORRES, PABLO GONZALEZ, MARGARITA L. ELICES, KOZABURO HAYASHI, and MANUEL ELICES
}

Depto de Ciencia de Materiales, Universidad Politecnica, E.T.S.I. Caminos, Canales y Puertos, c/ Profesor Aranguren s/n, 28040 Madrid, Spain; Laboratorio de Investigacion Cardiovascular, Departamento de Medicina y Cirugia Experimentales, Hospital Clinico Universitario San Carlos, 28040 Madrid, Spain; Departamento Anatomia Patologica, Clinica Ruber, 28006 Madrid, Spain; and Research Institute of Technology, Okayama University of Science, Okayama 700-0005, Japan

\begin{abstract}
Background This work explores for the first time the effects of temperature increments on the development of high shear stresses between plaque and arterial wall due to their different dilatational properties. Data from the literature report febrile reactions prior to myocardial infarction in patients with normal coronary arteries and that coronary syndromes seem to be triggered by bacterial and viral infections, being fever the common symptom. Methods The thermo-mechanical behavior of thoracic aortas of New Zealand White rabbits with different degrees of atherosclerosis was measured by means of pressure-diameter tests at different temperatures. In addition, specific measurements of the thermal dilatation coefficient of atheroma plaques and of healthy arterial walls were performed by means of tensile tests at different temperatures. Results Results show a different thermo-mechanical behavior, the dilatation coefficient of atheroma plaque being at least twice that of the arterial wall. The calculation of temperature-induced mechanical stress at the plaque-vessel interface yielded shear stress levels enough to promote plaque rupture. Conclusions Increases of corporal temperature either local-produced by the inflammatory processes associated with atherosclerosis-or systemic - by febrile reactions - can play a role in increasing the risk of acute coronary syndromes, and they deserve a more comprehensive study.
\end{abstract}

Keywords-Atherosclerosis, Rabbit model, Thermal dilatation coefficient, Shear stress.

\section{INTRODUCTION}

Acute coronary syndromes (ACS), which include unstable angina and acute myocardial infarction (MI), result from coronary thrombosis occurring at sites of plaque rupture or superficial erosion.

The events leading to plaque instability are not entirely known, but it is widely accepted that plaque cap weakening contributes to coronary plaque instability and vulnerability. The central lipid core of vulnerable atherosclerotic plaques accounts for more than $40 \%$ of the total volume lesion, and plaque caps are potentially vulnerable to mechanical stress and/or exacerbated inflammation. In addition, several other factors external to the coronary plaque such as vasoconstriction and high blood-pressure may increase the chance of rupture, and hypercoagulability at the sites of plaque rupture may cause thrombi formation, and subsequently, severe coronary occlusion.

Several prospective and retrospective studies have suggested that acute respiratory infection might trigger myocardial infarction, ${ }^{3}$ and that patients with MI had significantly more acute respiratory-tract infections in the 10 days before the index date of MI than the control subjects in the same time period. Recent clinical and experimental studies indicate that MI and stroke may be prevented by influenza vaccination, since this vaccination is negatively associated with the development of recurrent MI. Although some potential mechanisms and contributing factors have been proposed as relating influenza virus and bacterial infection to the development of ACS, the mechanism whereby a systemic viral or bacterial infection can induce plaque rupture or erosion remains unclear.

Fever is the common symptom shared by most respiratory bacterial and viral infections, and, interestingly, patients with angiographically normal coronary arteries suffering from MI frequently had febrile reactions prior to MI. The authors have demonstrated in a previous work a close relationship between mechanical stresses and temperature variations in the arterial wall, which in turn could lead to 
the development of highly stressed regions between materials with different dilatational properties.

On the basis of our previous results on the coupled thermo-mechanical behavior of blood vessels and the fact that fever is the common clinical symptom of both bacterial and viral infections, we explore in the present work the effect of increases of corporal temperature, and in particular the effect of the mismatch of the lineal dilatation coefficient of the arterial wall and atherosclerotic plaque on the development of interfacial shear stresses that can lead to plaque detachment and thrombi formation. In this study, the thermomechanical properties of arterial vessels with different degrees of atherosclerosis have been measured by means of a rabbit model, determining the different dilatational behavior of the atheromatous plaque and arterial wall. The mechanical stresses at the plaquevessel interface have been calculated by means of a simple model that gives the average shear stress. The paper closes by discussing whether, in addition to other mechanisms still under consideration, increases of corporal temperature can play a role in increasing the risk of ACS, and if they merit a more comprehensive study.

\section{METHODS}

\section{Material}

Experiments were conducted in accordance with the "European Convention on Animal Care" on male New Zealand White rabbits weighing $3.8-5.0 \mathrm{~kg}$. The descending thoracic aorta was excised from rabbits with different degrees of atherosclerosis induced by hypercholesterolemia.

Nineteen rabbits were used for the study. They were randomly sorted into three groups:

(a) Group A: Six rabbits were fed on a standard diet for 90 days. Aortas from this group were used as control.

(b) Group B: Eight rabbits were fed on a high-cholesterol diet (rabbit chow supplemented with $2 \%$ cholesterol, $100 \mathrm{~g} /$ day/animal) for a period of 45-55 days. This group developed hypercholesterolemia and atherosclerotic lesions, and was used to evaluate the medium-term development of the disease.

(c) Group C: Five rabbits were fed on the same $2 \%$ cholesterol-rich diet for a period of 90 days. Vessels from this group developed large atherosclerotic lesions and were used for the long-term evaluation.

After the corresponding period, all the animals were euthanized with an intravenous overdose of pentobarbital sodium and their thoracic aortas were harvested. Before excision, the in vivo reference length was marked by means of two suture loops. Collaterals, if any, were occluded by hemostatic clips (Ethicon Ligaclip MCA). Specimens were preserved in saline solution at $4{ }^{\circ} \mathrm{C}$ until testing within $12 \mathrm{~h}$ from excision. Although the short time after extraction ensured that muscle cells were alive no activation was induced in order to analyze the passive behavior of the arterial wall.

The clinical protocol, carried out in the Hospital Clinico San Carlos of Madrid, was conducted in compliance with the Directive 86/609/EEC of the European Union on the protection of animals used for experimental and other scientific purposes, and was approved by the hospital's ethics committee.

\section{Biochemical Measurements}

Plasma lipids (total cholesterol, HDL-cholesterol, and LDL-cholesterol) and IL-1/? levels were measured in rabbits from group A (control) at baseline, 45, 55, and 90 days after starting the standard diet. In group B as well as in group C, plasma lipids and IL-1/? were determined at baseline, 45, 55, and 90 days after starting the high-cholesterol diet. All biochemical measurements were performed in duplicate. Total plasma cholesterol (mg/dL), LDL-cholesterol (mg/dL), and HDL-cholesterol (mg/dL) were measured by colorimetric methods using commercial kits (Sigma Diagnosis Inc., St. Louis, USA).

\section{Histology}

The thoracic aortas were washed with sterile saline solution after removal from the body. Histological analysis was performed on $10 \mathrm{~mm}$ edge portions to determine the wall thickness and structure, and the size and morphology of the atherosclerotic lesions. Two rings from the aorta were stained with oil-red-O. The remaining tissue was fixed in $10 \%$ formalin in no-load condition for at least $24 \mathrm{~h}$. Fixed arteries were sectioned into 3-mm rings for embedding in paraffin and stained by hematoxylin/eosin and orcein to resolve the elastic fibers.

\section{Thermo-mechanical Pressure-Diameter Tests}

Quasi-static in vitro pressure-diameter tests were carried out at four different temperatures including the physiological, $17,27,37$, and $42{ }^{\circ} \mathrm{C}$, which encompass most situations of interest, from severe hypothermia for cardiac surgical procedures to febrile events.

Arterial segments, approximately $45 \mathrm{~mm}$ long and $6 \mathrm{~mm}$ diameter, were fixed to the grips of an electromechanical tensile testing machine (Instron 4411) by 
means of appropriate veterinary needles. A $100 \mathrm{~N}$ load cell (Instron 2525-806, precision $>0.1 \mathrm{~N}$ ) was used to monitor the axial force applied to the specimen. The lower grip was specially designed to allow internal pressurization of the vascular segment through the needle. The experimental device is sketched in Fig. 1.

During the tests, specimens were kept inside a transparent chamber and submerged in PBS solution heated by a thermostatic bath (Unitronic 6320200). The temperature of the vessel was controlled to $0.5{ }^{\circ} \mathrm{C}$ by a $\mathrm{K}$-type thermocouple located in the chamber and close to the artery $(<4 \mathrm{~mm})$.

Specimens were first stabilized at $37{ }^{\circ} \mathrm{C}$ and stretched to their in vivo length, which was equal, on average, to 1.2 times the length at rest. No significant differences were found in the in vivo length of control and atherosclerotic rabbits. Then, pressurization tests were sequentially performed on every specimen starting at the lowest temperature $\left(17^{\circ} \mathrm{C}\right)$. A stabilization period of at least $15 \mathrm{~min}$ was allowed at each test temperature. The average total test duration per sample (tests at the four temperatures) was $120 \mathrm{~min}$. To check reproducibility, some samples previously tested at the four temperatures $\left(17,27,37\right.$, and $\left.42{ }^{\circ} \mathrm{C}\right)$ were tested once again at $27{ }^{\circ} \mathrm{C}$. Since no appreciable differences were observed between pressure-diameter curves of the two tests at $27{ }^{\circ} \mathrm{C}$, it was concluded that pressure-diameter tests were not affected by the duration of the test.

After the stabilization period at the prescribed temperature, intravascular pressure was applied to the vessel via the lower needle. Pressure was measured with a transducer with $0.02 \mathrm{kPa}(0.15 \mathrm{mmHg})$ accuracy (Druck PMP 4000). Synchronized measurement of the outer diameter at the midpoint section was achieved using an optical extensometer with $1 / \mathrm{mi}$ accuracy (Keyence LS-7500).

Transmural pressure, i.e., the pressure difference between the inside and the outside of the vessel, was cycled from 0 to $26.66 \mathrm{kPa}(0-200 \mathrm{mmHg})$ at a loading rate of $0.4 \mathrm{kPa} / \mathrm{s}(3 \mathrm{mmHg} / \mathrm{s})$ until stress-relaxation effects were removed. A stable and reproducible response with a negligible hysteresis loop was achieved typically within five cycles-and always before ten-in all the specimens. After pre-conditioning, aortas were once more pressurized up to $26.66 \mathrm{kPa}(200 \mathrm{mmHg})$ at $0.4 \mathrm{kPa} / \mathrm{s}(3 \mathrm{mmHg} / \mathrm{s})$, and the loading branch was used for the analysis.

\section{Thermo-mechanical Tensile Tests}

Tensile tests at reference temperatures $(17,27,37$, and $42{ }^{\circ} \mathrm{C}$ ) were performed in order to characterize the

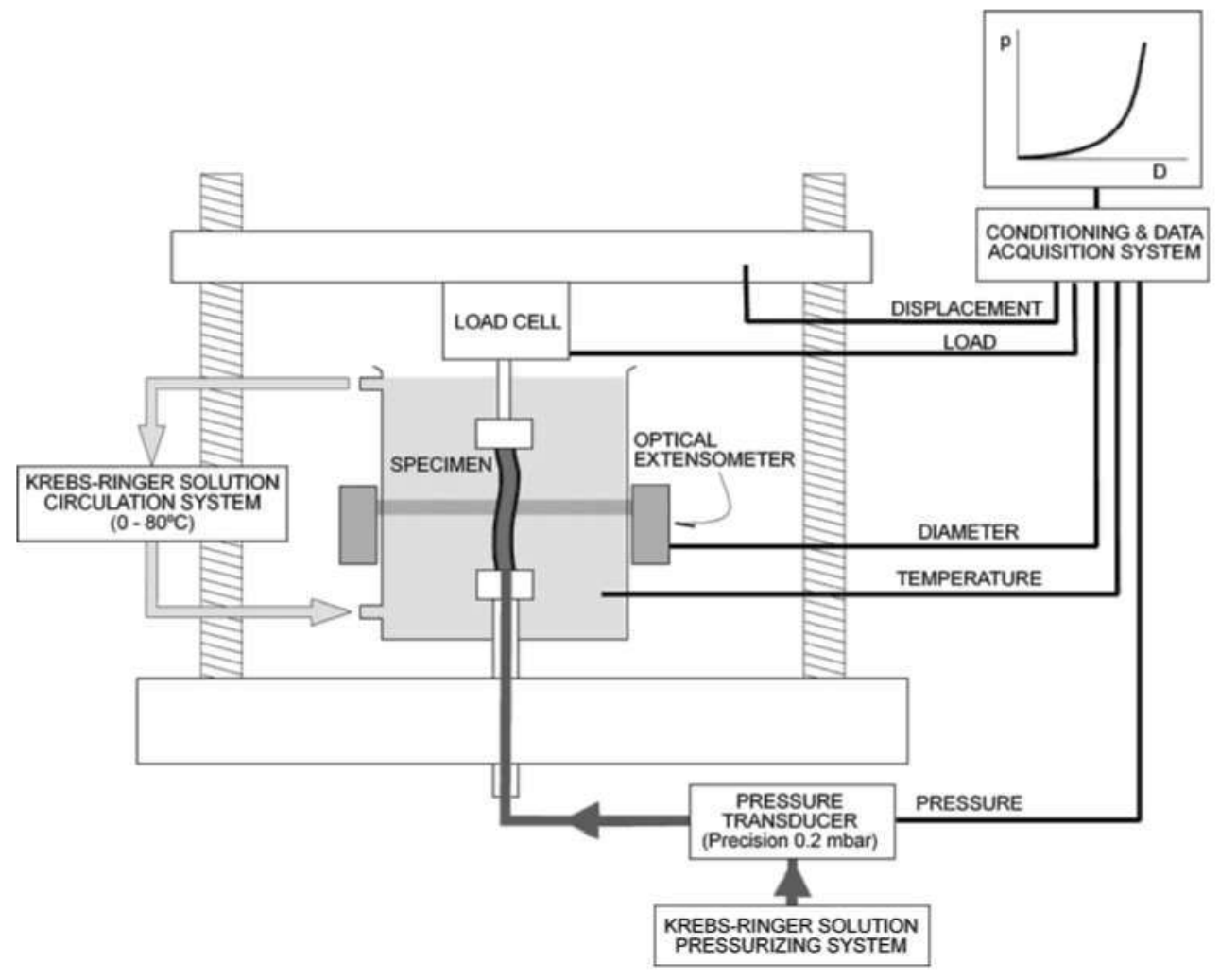

FIGURE 1. Experimental set-up for pressure-diameter tests. 
behavior of the healthy arterial wall and the plaque separately. Two types of specimens were tested:

- Healthy aortic wall specimens were obtained from arteries of group A. Nominal $10 \times 2 \mathrm{~mm}^{2}$ strips were obtained from longitudinally cut opened arteries. Specimens were excised with their longitudinal axis coincident with the circumferential direction of the vessel.

- Atheromatous plaques averaging $10 \mathrm{~mm}$ in length and $4 \mathrm{~mm}$ in width were obtained from arteries of group $\mathrm{C}$ by gently removing the fibrous portion of vascular tissue. A blunt blade was used to avoid damaging the specimens. Standard $10 \times 2 \mathrm{~mm}^{2}$ tensile samples were obtained from plaque portions.

Specimens were fixed to the grips of an electromechanical tensile testing machine (Instron 4411) and placed in a thermostatic bath of PBS solution, as described for pressure-diameter tests. The axial force was measured with the tensile testing machine by means of a $10 \mathrm{~N}$ load cell (Instron 2519-101, precision > $5 \mathrm{mN}$ ) and the elongation by the internal LVDT sensor (precision > $10 / \mathrm{im}$ ). The crosshead displacement was taken as a direct measurement of the sample elongation, since the compliance of the sample had been estimated as 100 times that of the equipment. An optical extensometer with 1 fim accuracy (Keyence LS-7500) was used for continuous monitoring of the specimen thickness.

Unloaded specimens were stabilized at each test temperature for at least $15 \mathrm{~min}$. Then a few load cycles between 0 and $0.1 \mathrm{~N}$ were applied until a reproducible response with a negligible hysteresis loop was achieved. Specimen thickness was recorded at the beginning of the last cycle, in zero load condition. The ascending branch of the last cycle was used to compute the loaddisplacement curve of the specimen.

\section{Statistical Analysis}

Values within groups were averaged and expressed as mean \pm standard error. Unpaired Student /-test was used for comparison between groups. Differences were considered significant at probability P-values under 0.05 .

\section{Thermo-mechanical Analysis}

The effect of temperature was evaluated through the pressure-diameter and tensile curves measured at different temperatures. These curves were used to compute the thermal dilatation coefficient a of the aortic wall at different pressure and stress levels.
The thermal dilatation coefficient measures the change in size caused by temperature. Positive values correspond to substances that expand when heated, while negative ones indicate that the material shrinks as the temperature increases. In first order, the fractional variation in size is proportional to the temperature increment, and it is given by:

$$
d H / H=a c l T
$$

$\mathrm{x}$ being the thermal dilatation coefficient, $H$ the size, and $d H$ and $d T$ the infinitesimal variations of size and temperature. Integration of Eq. (1) between a reference state $\left(\mathrm{T}_{\mathrm{Ref}}, H_{R e l}\right)$ and $(T, H)$ yields $H=H_{R e t} e^{a\left({ }_{-} T_{-} T_{\mathrm{TM}^{\prime}}\right.}$ that can be rearranged ${ }^{12}$ :

$$
H=H^{*} e^{a T}
$$

where $H^{*}=H_{R e} f e^{a, T R e t}$ is a constant. The thermal dilatation coefficient a is readily obtained by direct fitting of this equation to the appropriate experimental data, irrelevant to $H_{r e t}$ or $T_{r e t}$ values. In the pressurediameter tests, the input for Eq. (2) were the pairs temperature-diameter $(T, D)$ measured at a given pressure. In tensile tests, the specimen length $L$ at a given stress level was chosen for the fit along with temperature $T$.

\section{RESULTS}

\section{Biochemical Determinations}

The values of plasma lipids for the three groups are shown in Table 1 . Over the 90 days plasma lipids did not change in control rabbits (group A, Table la) whilst a statistically significant increase over its baseline values is seen at 45 and 55 days for group B, and 90 days for group $\mathrm{C}$.

\section{Histology}

Figure 2 shows two images obtained with light microscopy from a healthy rabbit from control group A (Fig. 2a), and from a specimen of group B (Fig. 2b). No signs of atherosclerosis were observed in control aortas, whereas atheromatous plaques similar to that depicted in Fig. 2b were noticed in vessels from groups $\mathrm{B}$ and $\mathrm{C}$. In all cases, the arterial wall had a large amount of elastic fiber, with no remarkable difference between groups. Table 2 summarizes the histological measurements of the thickness of arterial wall and atheroma plaques. While atheroma plaques of the intimal layer develop and grow with the progression of the disease, arterial walls do not thicken significantly.

Lipids did not accumulate in the intima of rabbits from group A (control rabbits). For group B rabbits, 
TABLE 1. (a) Biochemical variables in control animals (group A) and (b) Long-term total cholesterol, HDL-cholesterol, LDL-cholesterol, and IL-1/J for rabbits from groups B and C.

(a)

$\begin{array}{lllll} & \text { Baseline } & 30 \text { days } & 90 \text { days } & \text { P-value } \\ \text { Total cholesterol } & 50 \pm 22 & 55 \pm 20 & 59 \pm 30 & \text { NS } \\ \text { HDL-cholesterol } & 20 \pm 7 & 21 \pm 8 & 25 \pm 7 & \text { NS } \\ \text { LDL-cholesterol } & 46 \pm 23 & 43 \pm 22 & 40 \pm 23 & \mathrm{NS} \\ \text { IL-1/? }(\mathrm{pg} / \mathrm{mL}) & 6.8 \pm 3.3 & 8.7 \pm 2.8 & 8.1 \pm 1.1\end{array}$

(b)

$\begin{array}{lcccc} & \text { Baseline } & 45 \text { days } & 55 \text { days } & 90 \text { days } \\ \text { Total cholesterol } & 51 \pm 30 & 2009 \pm 612^{*} & 1812 \pm 315^{*} & 1610 \pm 211^{*} \\ \text { HDL-cholesterol } & 22 \pm 8 & 410 \pm 66^{*} & 372 \pm 87^{*} & 374 \pm 72^{*} \\ \text { LDL-cholesterol } & 40 \pm 22 & 1212 \pm 195^{*} & 1600 \pm 232^{*} & 1501 \pm 312^{*} \\ \text { IL-1/? }(\mathrm{pg} / \mathrm{mL}) & 5.7 \pm 4.3 & 12 \pm 6.7^{*} & 32 \pm 9.8^{*} & 42 \pm 11.3 *\end{array}$

Plasma lipids are expressed as $\mathrm{mg} / \mathrm{dL}$.

'Statistically significant differences over controls.
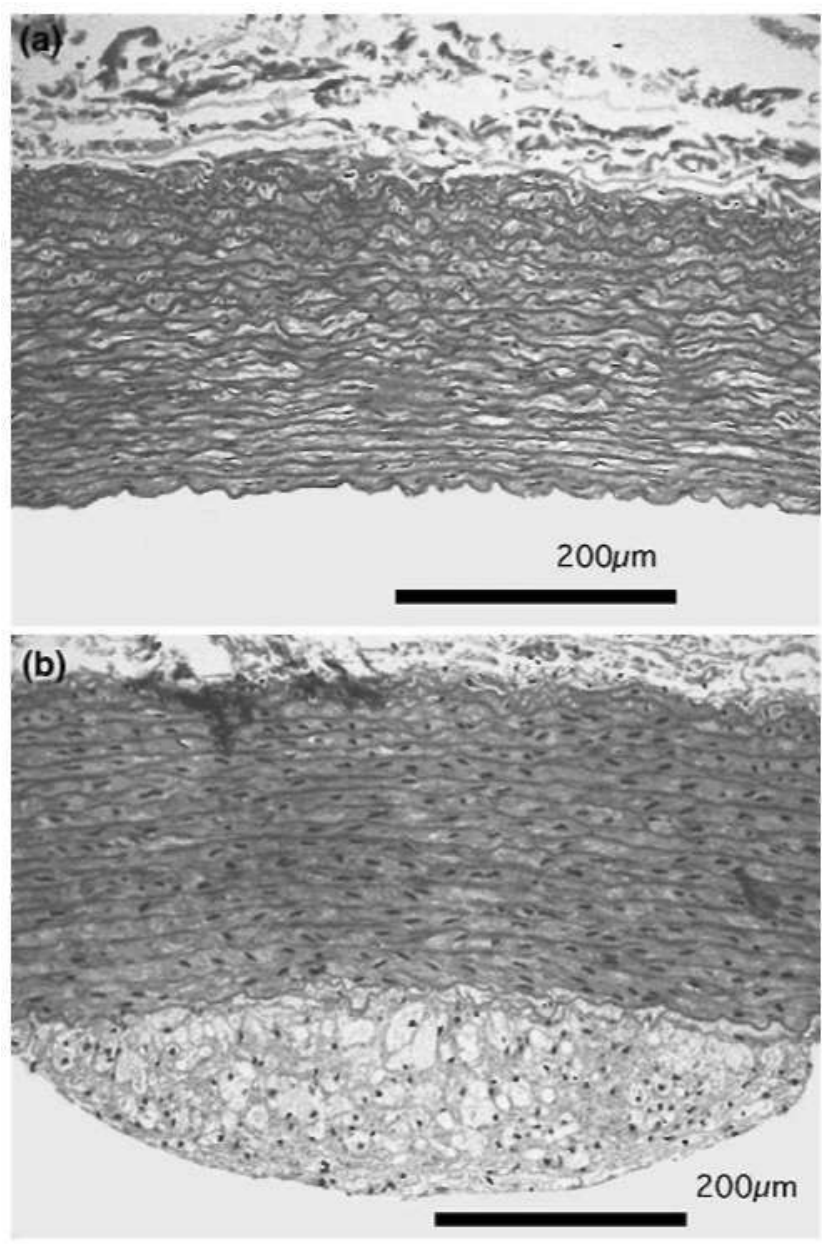

FIGURE 2. (a) Cross section of a healthy thoracic aorta from a control rabbit (group A), (b) Cross section of a thoracic aorta from an atherosclerotic rabbit (group B).
TABLE 2. Arterial wall and plaque thickness (Mean : Std. Dev.).

\begin{tabular}{lcccc}
\hline Group & $\begin{array}{c}\text { Days of } \\
\text { cholesterol-rich } \\
\text { diet }\end{array}$ & $\begin{array}{c}\text { Wall } \\
\text { thickness } \\
(\mathrm{mm})\end{array}$ & $\begin{array}{c}\text { Plaque } \\
\text { thickness } \\
(\mathrm{mm})\end{array}$ & $\begin{array}{c}\text { Plaque/ } \\
\text { Wall } \\
\text { ratio }\end{array}$ \\
\hline A & 0 & $0.26 \pm 0.02$ & - & 0 \\
B & 45 & $0.29 \pm 0.01$ & $0.09 \pm 0.01$ & 0.31 \\
B & 55 & $0.29 \pm 0.01$ & $0.22 \pm 0.02$ & 0.76 \\
C & $>70$ & $0.28 \pm 0.01$ & $0.34 \pm 0.015$ & 1.21 \\
\hline
\end{tabular}

oil-red-O staining excluded lipid accumulation in the intima at 30 days but at 45 and 55 days macroscopic lesions were grossly visible and oil-red-O staining showed lipid accumulation in the intima (Fig. 2b). Soft atheroma plaques with high lipid content were formed in rabbits of groups $\mathrm{B}$ and $\mathrm{C}$, as well described in the literature.

\section{Pressure-Diameter Tests}

Mean pressure-diameter curves of thoracic arteries measured at 17, 27, 37, and $42{ }^{\circ} \mathrm{C}$ are shown in Fig. 3. Values of mean diameters and standard errors at $16 \mathrm{kPa}(120 \mathrm{mmHg})$ inner pressure are given in Table 3. Pressure-diameter curves show two relatively well-defined portions below and above a pressure level of about $p_{Q}=10.67 \mathrm{kPa}(80 \mathrm{mmHg})$. The first part of the curves, under $p_{Q}$, is more compliant and less sensitive to changes due to atherosclerosis and temperature. The second part-above $p_{Q}$-is steeper and depends markedly on the stage of the atherosclerotic disease and the test temperature. 


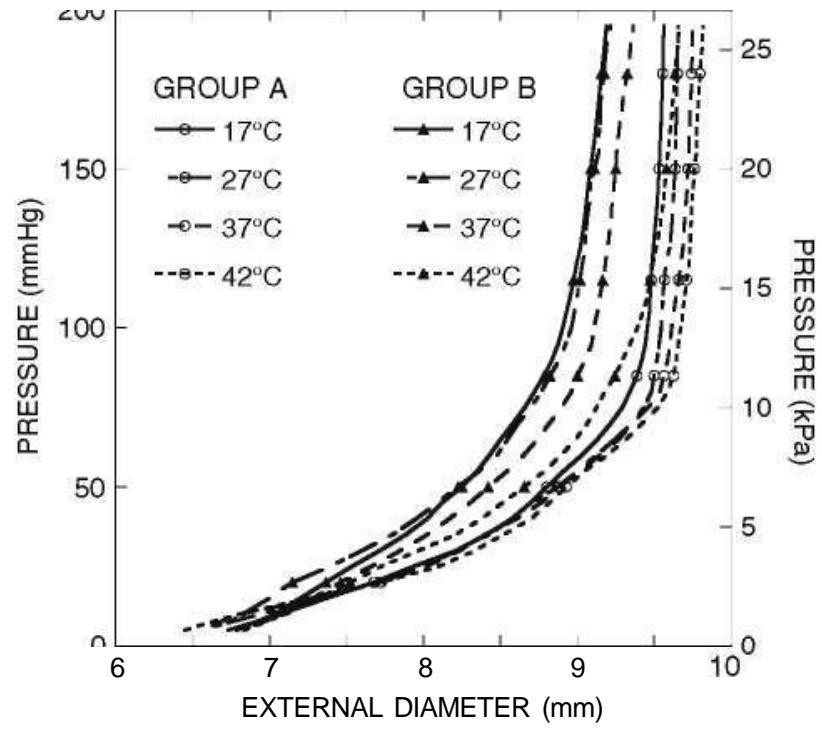

FIGURE 3. Mean pressure-diameter curves of the thoracic aortas at the four test temperatures. Group A: healthy control group. Group B: 50 days average cholesterol-rich diet.

TABLE 3. Thoracic aorta diameters at $16 \mathrm{kPa}(120 \mathrm{mmHg})$ pressure level.

Diameter $(\mathrm{mm})$ at $\mathrm{p}=16 \mathrm{kPa}(120 \mathrm{mmHg})($ Mean \pm Std. Err.)

\begin{tabular}{lcccc} 
Group & $17{ }^{\circ} \mathrm{C}$ & $27{ }^{\circ} \mathrm{C}$ & $37{ }^{\circ} \mathrm{C}$ & $42{ }^{\circ} \mathrm{C}$ \\
\hline A & $9.5 \pm 0.7$ & $9.6 \pm 0.6$ & $9.7 \pm 0.6$ & $9.7 \pm 0.6$ \\
B & $9.0 \pm 0.6$ & $9.2 \pm 0.6$ & $9.0 \pm 0.6$ & $9.5 \pm 0.6$ \\
C & $8.5 \pm 1.5$ & $8.7 \pm 1.5$ & $9.0 \pm 1.6$ & $9.4 \pm 1.8$ \\
\hline
\end{tabular}

Group C vessels no longer maintained a cylindrical configuration and were used mainly as a source of atheroma plaques for tensile tests. Pressure-diameter curves for atherosclerotic vessels-group C-showed an atypical behavior due to the inhomogeneities on the arterial wall produced by atheroma plaques and their curves are not displayed in Fig. 3. For comparison purposes, their mean diameters at $p=16 \mathrm{kPa}$ $(120 \mathrm{mmHg})$ are shown in Table 3.

At a given temperature, it is observed a progressive stiffening of the vessels, indicating the effects of the advance of atherosclerosis. The most compliant arteries were the healthy ones from control group A. Temperature effects were greater in atherosclerotic vessels, where artery diameters changed noticeably with temperature at a given pressure.

The thermal dilatation coefficient a was computed by least square fitting of Eq. (2) to temperaturediameter pairs measured at the same pressure level. Figure 4 shows the mean value of a and the standard error as a function of pressure. Healthy and

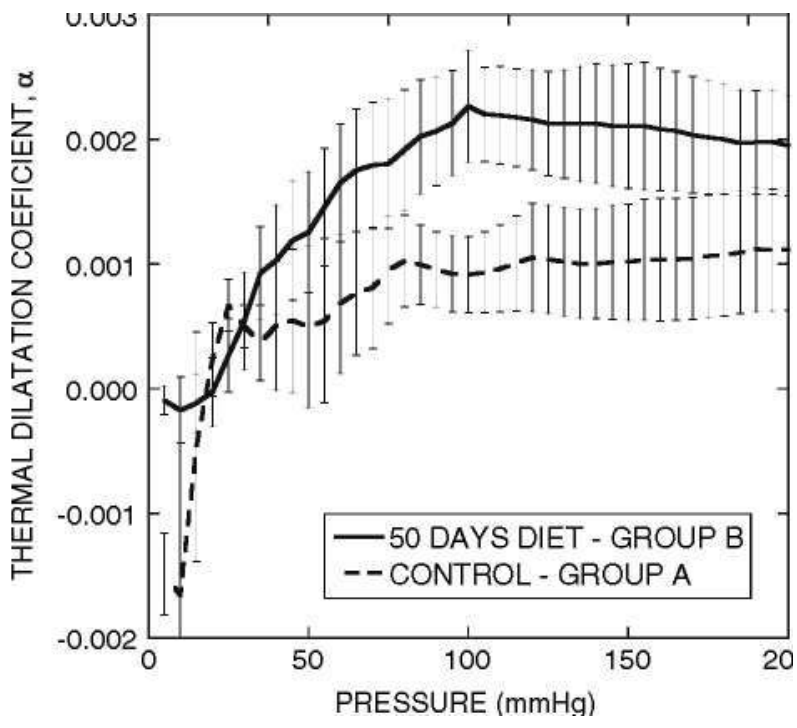

FIGURE 4. Mean thermal dilatation coefficient and standard error deduced from pressure-diameter tests. Group A: healthy control group. Group B: 50 days average cholesterol-rich diet.

medium-term diseased thoracic arteries-groups A and $\mathrm{B}$, respectively-displayed an inversion of the thermal dilatation coefficient: negative values (indicating that arterial wall diameters shrink when heated) were obtained at low pressure while positive $\mathrm{x}$ were found at pressures over $1.33-2.66 \mathrm{kPa}$ $(10-20 \mathrm{mmHg})$. The thermal dilatation coefficient increased monotonically with pressure level with a progressively decreasing slope, approaching a saturation value at physiological pressures.

\section{Tensile Tests}

Specific tensile tests were performed to compare the behavior of the healthy arterial wall and the plaque separately. Healthy aortic wall (obtained from vessels of group A) and atheromatous plaque (obtained from arteries of group C) were tested.

Figure 5 shows the tensile curves obtained for both types of tissues at $37{ }^{\circ} \mathrm{C}$. Stress $a$ was computed by dividing force $F$ by the initial cross-sectional area $A_{Q}$, and the strain is represented by the stretching $X$ that was obtained by dividing the instantaneous length $L$ by the initial length $L_{Q}$. The degree of variability of curves in Fig. 5 is comparable to other studies and attributable to arterial wall inhomogeneities.

In order to measure the thermal dilatation coefficient of atheroma plaques, five tensile specimens obtained from group $\mathrm{C}$ aortas were tested at 17, 27, 37, and $42{ }^{\circ} \mathrm{C}$. The variation of specimen length with 


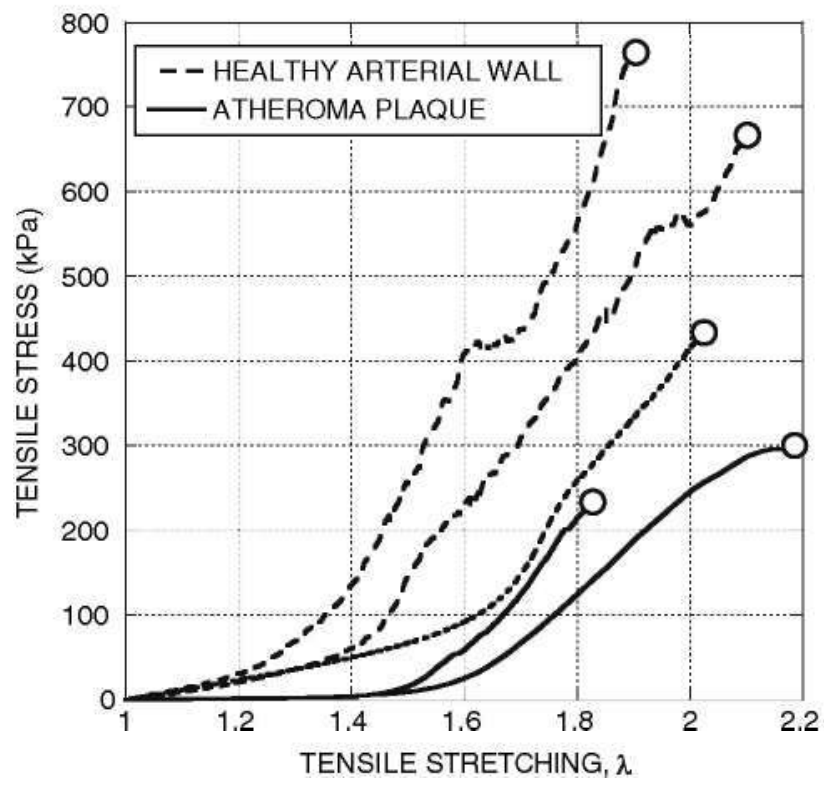

FIGURE 5. Tensile curves of healthy arterial wall from rabbits of control group $A$, and atheromatous plaque obtained from rabbits from group C (90 days of cholesterol-rich diet). Test temperature $37^{\circ} \mathrm{C}$.

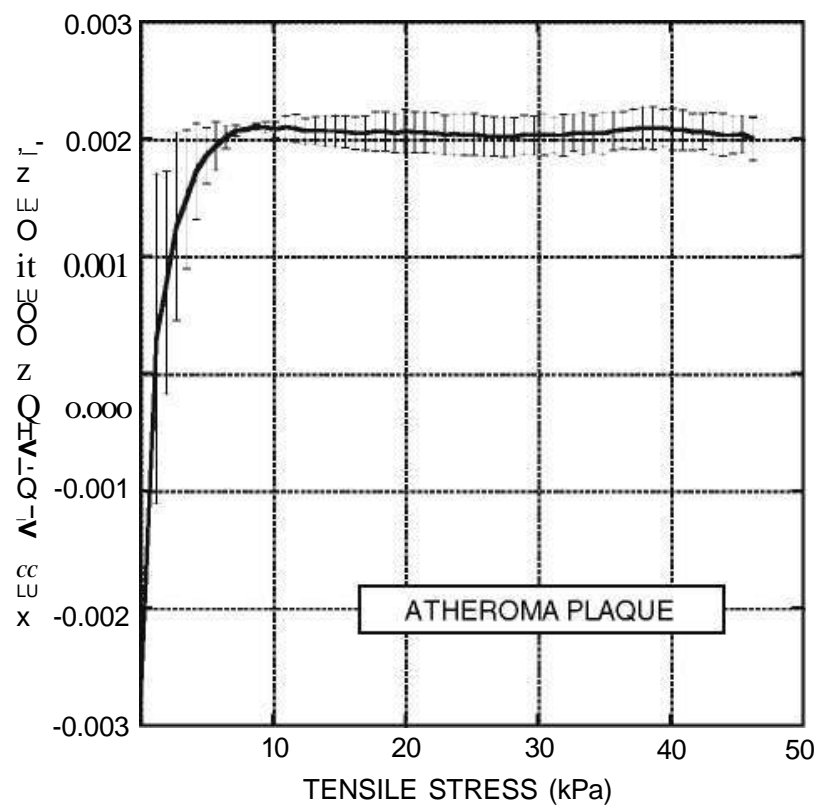

FIGURE 6. Thermal dilatation coefficient of atheromatous plaque obtained from tensile tests at $17,27,37$, and $42{ }^{\circ} \mathrm{C}$. Mean values and standard deviations are shown.

temperature at a given stress level was used to compute the thermal dilatation coefficient by fitting of Eq. (2) to temperature-length pairs, as described above. The mean values of the resulting thermal dilatation coefficients are plotted in Fig. 6 along with their standard errors.

\section{DISCUSSION}

This work shows for the first time that the increment of temperature has an influence on the development of high shear stresses between plaque and arterial wall due to their different thermal dilatational properties. This sheds light on the role of mechanical stresses in plaque instability since results from the literature suggest that plaque rupture is not well characterized by considerations based on plaque nominal strength or critical stresses alone. Although the rabbit model is unable of reproducing the advanced atherosclerotic plaque with large lipid-core and a thin fibrous cap, characteristic of advanced human lesions, it is valuable to assess the response to temperature increments and stability of small atheromatous plaques, clinically silent, that may be at the root of a large number of MI.

Thoracic aortas of New Zealand White rabbits with different degrees of atherosclerosis induced by hypercholesterolemia were considered. The biochemical and histological analyses (Tables 1 and 2, and Fig. 2) clearly show the grade of development of the atherosclerosis, which can be related either to the plaque thickness or to the plasma lipid levels. The lipid accumulation in the intima is observed in advanced stages of the illness (group C) and plaque development becomes evident. Lesions type II III according to American Heart Association criteria were formed, as shown in Fig. 2. IL-1/? levels (Table 1) show that, as in human patients, atheromatous plaques in rabbits are connected to inflammatory processes.

Pressure-diameter curves reflect the progress of illness (Fig. 3). Curves become stiffer (specially in its second part, over $10.67 \mathrm{kPa}(80 \mathrm{mmHg})$ ), and eventually show non-homogeneous behavior due to plaque formation, as in group $\mathrm{C}$. These results agree with previous works, that show that the arterial wall stiffens with the development of atherosclerosis. Nevertheless, tensile tests on atheroma plaques revealed that these tissues are more compliant than healthy arterial wall by at least one order of magnitude (Fig. 5). These findings are in very good accordance with a previous work by Hayashi and coworkers. A possible explanation of the higher stiffness of pressurediameter measurements is the stiffening effect due to the multiaxial loading present in the pressure-diameter test due to the axial stretching, and the fact that atherosclerosis is accompanied by a thickening of the arterial wall. In addition, the modulus of elasticity of the diseased arterial wall does not change noticeably unless considerable calcification in the wall is present. As a result, prediction of atherosclerosis based on stiffness measurements from pressure-diameter curves is an involved problem of uncertain application to clinics. 
The effect of temperature is markedly higher on pressure-diameter curves of atherosclerotic vessels (groups B and C) than on healthy ones (group A): For a given temperature increment (e.g., from 37 to $42{ }^{\circ} \mathrm{C}$ ), pressure-diameter curves differ more pronouncedly as atherosclerosis develops (Fig. 3 and Table 3). The observed dependence suggests that atherosclerosis induces a significant variation of the thermal dilatation coefficient a, a parameter that accounts for the dilatational properties of the arterial wall. Subsequently, a large difference is expected between the thermal dilatation coefficient of the healthy arterial wall and the diseased one.

The variation of the thermal dilatation coefficient derived from pressure-diameter tests depends significantly on the degree of atherosclerosis as is shown in Fig. 4. Healthy rabbits (group A) show the typical behavior of arterial wall with negative values in the no load condition, an inversion point at «1.33$2.66 \mathrm{kPa}(10-20 \mathrm{mmHg})$, and a positive plateau at physiological pressures $(-13.33 \mathrm{kPa}(100 \mathrm{mmHg}))$ and above $\left(\mathrm{a}=+1 \times 10^{\prime \prime 3}\left({ }^{\circ} \mathrm{C}\right)^{-1}\right)$. This closely matches a previous work on human carotid arteries by the authors. ${ }^{12}$ Medium-term diseased vessels, group B, show an increment in a values of up to $+2 \times 1 \sim^{3}$ $\left({ }^{\circ} \mathrm{C}\right)^{-1}$ with a dependence on pressure level resembling that from healthy vessels of group A. The inversion point is maintained at $1.33-2.66 \mathrm{kPa}(10-20 \mathrm{mmHg})$.

A specific measurement of the thermal dilatation coefficient of atheroma plaques can be performed by tensile tests performed at different temperatures, as explained in sections "Methods" and "Results." The resulting coefficient is about $+2 \times 10 \sim^{3}\left({ }^{\circ} \mathrm{C}\right)^{-1}$, and shows an inversion point at low stresses (Fig. 6). This $a$ duplicates the value of arterial wall $\left(+1 \mathrm{x} 10^{\prime \prime 3}\left({ }^{\circ} \mathrm{C}\right)^{-1}\right)$ obtained from healthy aortas.

Large differences in the thermal dilatation coefficient of arterial wall and atheromatous plaque can lead to high interfacial stresses that could eventually provoke plaque detachment in response to a temperature increment. The temperature increment produced by the inflammatory processes associated with atherosclerosis can raise the local temperature of the plaque by up to $2{ }^{\circ} \mathrm{C}$, and temperature differences between atherosclerotic plaque and healthy vessel wall of over $0.5{ }^{\circ} \mathrm{C}$ are a predictor of adverse cardiac events in patients undergoing successful percutaneous intervention. In addition, febrile reactions add up to local temperature increments, placing hyperthermia as a contributing factor to ACS that has to be taken into account.

When two layers of materials with different thermal properties are bonded together, temperature increments generate interfacial shear stresses that develop near the ends, typically along a distance of the same order of magnitude as the thickness of the layers. The exact distribution of shearing stress along the interface cannot be determined in an elementary way, and some hypotheses about the interface have to be made.

A simple thermo-elastic model was devised to assess the effect of temperature changes on the development of shear stresses at the plaque-vessel interface. The model is inspired by the classical work by Kelly and Tyson on fiber reinforced materials, and assumes a constant interfacial shear stress.

Figure 7 shows the model geometry, where the vulnerable plaque is represented by a layer of length $L$ and thickness $B$ placed on a substrate. The linear dilatation coefficient of plaque and substrate are op and $\mathrm{a}_{\mathrm{s}}$, respectively, and no thermal or residual stresses are considered at the reference temperature. It is assumed in the following that $a_{p}>a_{s}$, as was found in the tests previously described.

Shear stresses arise at the interface under a temperature increment $A T$, as a consequence of the different thermal strains of the plaque $\left(a_{p} A 7\right)$ and substrate $\left(a_{s} A 7\right)$. The plaque tends to increase in length by a proportion of $\left(a_{p} A 7\right)$, and is hindered by the smaller extension ratio of the substrate $\left(a_{s} A 7\right)$.

If constant shear stresses $\mathrm{x}_{0}$ develop at lengths $L^{*}$ at both ends of the plaque, the maximum longitudinal compressive stress $<_{\mathrm{m}}$ on the plaque is computed as (Fig. 7):

$$
f f m=\boldsymbol{B} \boldsymbol{J}_{0}^{\mathrm{xdx}}=-j \boldsymbol{j}-
$$

The strain in the central portion of the plaque is the sum of the thermal dilatation $a_{p}$ ATplus the shortening caused by the compressive stress, equal to:

$$
\text { « } \mathrm{p} A \mathrm{~T}-\wedge
$$

$E_{p}$ being the modulus of elasticity of the plaque. Given that the plaque stiffness $\left(B E_{p}\right)$ is at least one order of magnitude less than that of the substrate (as experimental results from this work and by other authors suggest ${ }^{15}$ ) we can assume that the strain in the central portion of the plaque is, in first order approximation, equal to the thermal strain imposed by the substrate $\mathrm{x}_{\mathrm{s}}$ Ar. This leads to:

$$
o c_{s} A T=\quad a_{p} A T--^{\wedge}=\text { op } \mathrm{AT}-
$$

from which we obtain the shear stress as:

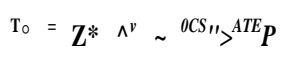

and if we realize that for geometrical reasons $L^{*}<L / 2$, we arrive at: 

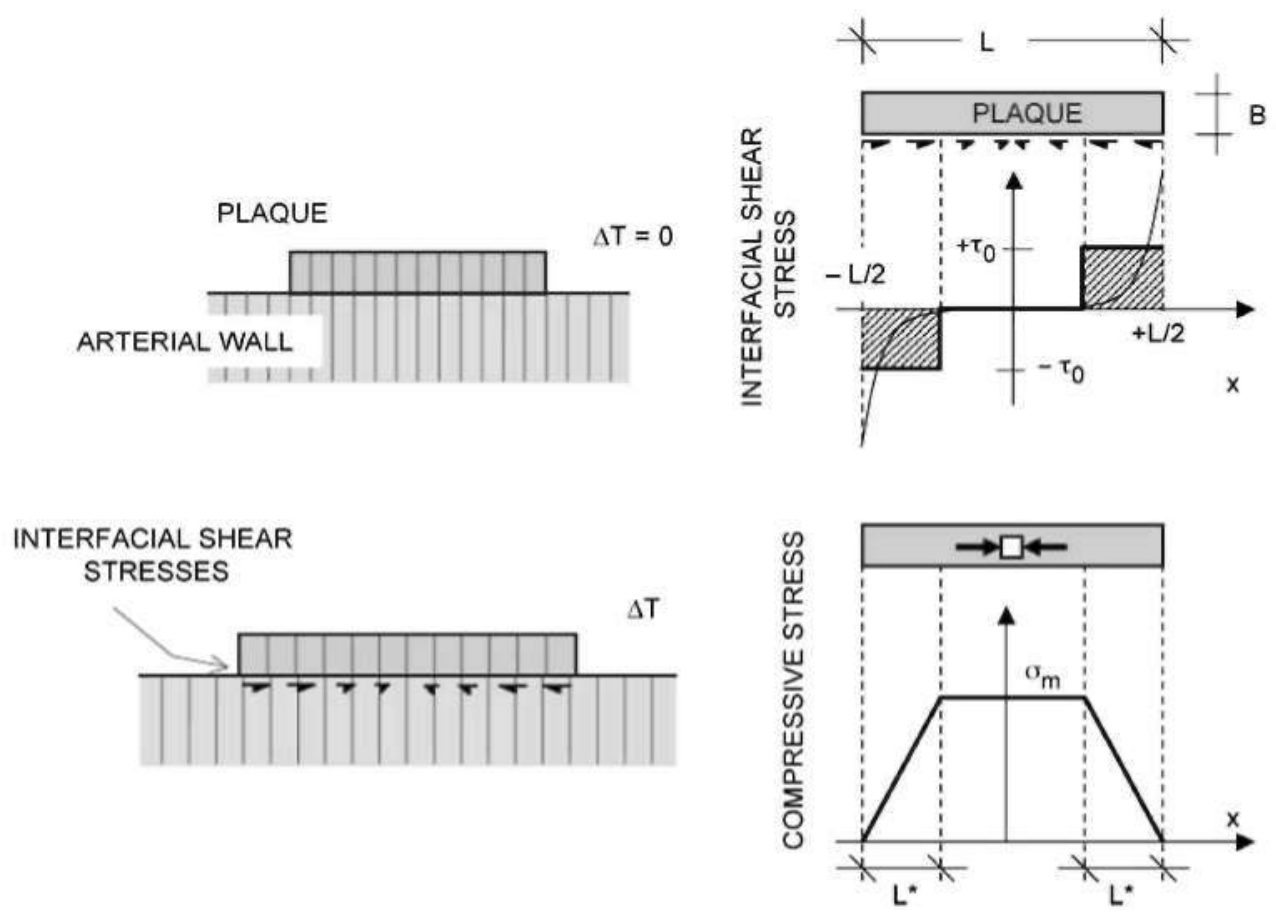

FIGURE 7. Model for stress analysis of plaque-vessel thermal interaction. Shear stresses develop at the interface between plaque and arterial wall, inducing a compressive stress state in the plaque.

$$
\mathrm{x}_{0}>2-\left(\text { ap }-a_{s}\right) A T E_{p}
$$

an equation that gives a lower bound of the interfacial shear stress.

The stress $\mathrm{x}_{0}$ considered above can be used as a conservative appraisal of the average shear stresses developed at the interface, which in general attains their maximum values at the ends of the interfacial zone and quickly drops to zero at a distance of the order of plaque thickness, as shown in Fig. 7.

The variation of shear stresses along the plaque can be determined by means of some other well-known interfacial models , although unfortunately the maximum shear stress is highly dependent on the specific assumptions made for the interface. For example, the model by Wang can be shown to provide a shear stress profile given by:

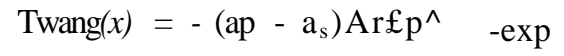

where $/ z_{a}$ is the thickness of the interfacial layer placed between plaque and substrate. Equation (8) is obtained under the assumption that all the layers are incompressible and that the elastic modulus of the interface is comparable to that of the plaque. The maximum stress predicted by Eq. (8) depends critically on the ratio $B / h^{\wedge}$, and tends to infinity when $/ \mathrm{z}_{\mathrm{a}}$ tends to zero. The mean shear stress computed from Eq. (8) provides:

$$
\begin{aligned}
\text { MWang }_{\text {W }} \int_{0}^{L / 2} \tau d x= & 3 \frac{B}{L}\left(\alpha_{\mathrm{p}}-\alpha_{\mathrm{s}}\right) \Delta T \\
& \times E_{\mathrm{p}}\left[1-\exp \left(\frac{-L}{2 \sqrt{B h_{\mathrm{a}}}}\right)\right]
\end{aligned}
$$

and for a thin interface $\left(y / B h_{\&}<\mathrm{C} L\right)$ this equation leads to:

$$
\mathbf{M}_{\text {Wang }} \quad 3-\left(\mathrm{a}_{\mathrm{p}}-\mathrm{a}_{\mathrm{s}}\right) \operatorname{Ar} £_{\mathrm{p}}
$$

a value comparable to that given by Eq. (7).

A similar result can be obtained from the shear lag model applied to the geometry shown in Fig. 7. In this case, the mean shear stress is:

$$
\left({ }^{\mathrm{T}}\right) \operatorname{cox}=i \quad / \quad \mathrm{T} \operatorname{rfx}=2-\left(\ll \mathrm{p}-a_{s}\right) A T
$$

\section{cosh $\boldsymbol{k} \boldsymbol{h}$}

$K$ being a constant related to the interfacial stiffness. Noting that when the plaque is firmly bonded to the substrate is $K L^{l} \$ E_{v}$ and then $1-1 / \cosh \left(J K / E_{V} \cdot L / 2\right) \ll 1$, Eq. (11) yields: 


$$
\left({ }^{\mathrm{T}}>\operatorname{Cox} \sim{ }^{2} \wedge\left({ }^{\mathrm{a}} \mathbf{p}-{ }^{\mathrm{a}} \mathrm{S}\right){ }^{\mathrm{A} r} £ \mathrm{p}\right.
$$

in agreement with Eq. (7).

The interfacial shear stress level between the atheromatous plaque and the arterial wall originated by variations of temperature has been assessed by firstorder approximation using Eq. (7). Assuming a dilatation coefficient of $+1 \times 10^{\prime 3^{3}}\left({ }^{\circ} \mathrm{C}\right)^{-1}$ for the arterial wall, $+2 \times 10 \sim^{3}\left({ }^{\circ} \mathrm{C}\right)^{-1}$ for the atheromatous plaque (both under normal physiological pressures), and rough value of $E_{p}$ equal to $600 \mathrm{kPa}$ (deduced from the tests on atheromatous plaques shown in Fig. 5 by averaging their slopes), the mean shear stresses developed at the plaque-vessel interface are:

$$
\mathrm{x}_{0}>2-\left(\mathrm{op}-a_{s}\right) A T E_{p}=1.2 \mathrm{kPa}\left({ }^{\circ} \mathrm{C}\right)^{-1} A T(B / L)
$$

which for $B / L \ll 0.2$ (a figure based on our measurements) and $A T=4{ }^{\circ} \mathrm{C}$ yields $\mathrm{x}_{0}>960 \mathrm{~Pa}$. This stress level is much higher than shear stresses due to blood flow (about 1-3 Pa) and it is considered enough to promote the detachment of the plaque.

In other words, the results of this work show that an increase of corporal temperature generates shear stresses between the plaque and the arterial wall that could be much higher than those produced by normal blood flow. In addition, the fact that the atheromatous plaque has a higher dilatation coefficient than the arterial wall generates a compressive stress state that increases plaque instability, leading eventually to the buckling of this structure.

Fatigue may be one of the mechanisms that might play a role in plaque rupture; fatigue is induced by repetitive loading, and is a symptomatically quiescent but potentially progressive failure process, which can result in sudden fracture at stress levels much lower than the critical stress. Moreover, repeated febrile processes give rise to additional large fatigue loads that can increase the risk of plaque debonding.

The formation of a platelet-rich thrombus takes place at the site of plaque disruption, but not all ruptured plaques result in thrombosis. In fact, postmortem studies in patients with ACS revealed that on most occasions thrombi are made of platelets and fibrin or composed of layers of different age, suggesting a gradual formation and recurrences of weak thrombogenic stimuli. This implies that subjects may be frequently exposed to potential triggers and that the levels of biochemical markers can be increased without an inevitable acute morbid event.

In this context, increases in corporal temperature would not be necessarily accompanied by an adverse event, but could contribute to the progressive weak- ening of the atheromatous plaque, making it prone to rupturing under lower levels of mechanical stress and/ or other triggering agents.

\section{ACKNOWLEDGMENTS}

The authors gratefully acknowledge financial support for this work provided by the Ministerio de Ciencia y Tecnologia (Spain) through projects MAT 2003-04906 and MAT 2005-06320, and by the Comunidad de Madrid through program ESTRUMAT-CM (reference MAT/77).

\section{REFERENCES}

Ammann, P., S. Marschall, M. Kraus, L. Schmid, W. Angehrn, R. Krapf, and H. Rickli. Characteristics and prognosis of myocardial infarction in patients with normal coronary arteries. Chest 117:333-338, 2000.

Bailey, J. M. Elasticity and tensile strength of normal and atherosclerotic rabbit aorta. /. Atheroscler. Res. 5:112-119, 1965.

Bainton, D., G. R. Jones, and D. Hole. Influenza and ischaemic heart disease - a possible trigger for acute myocardial infarction? Int. J. Epidemiol. l:Ti -2i9, 1978.

Bank, A. J., A. Versluis, S. M. Dodge, and W. H. Douglas. Atherosclerotic plaque rupture: A fatigue process? Med. Hypotheses 55:480-484, 2000.

Casscells, W., B. Hathorn, M. David, T. Krabach, W. K. Vaughn, H. A. McAllister, G. Bearman, and J. T. Willerson. Thermal detection of cellular infiltrates in living atherosclerotic plaques: possible implications for plaque rupture and thrombosis. Lancet 347:1447-1451, 1996.

Cox, H. L. The elasticity and strength of paper and other fibrous materials. Br. J. Appl. Phys. 3:72-79, 1952.

Davies, M. J. Stability and instability: two faces of coronary atherosclerosis. The Paul Dudley white lecture 1995. Circulation 94:2013-2020, 1996.

Falk, E. Why do plaques rupture? Circulation $86: 11130^{\wedge}-2$, 1992.

Fayad, Z. A., and V. Fuster. Clinical imaging of the highrisk or vulnerable atherosclerotic plaque. Circ. Res. 89:305-316, 2001.

Gibson, C M., L. Diaz, K. Kandarpa, F. M. Sacks, R. C. Pasternak, T. Sandor, C Feldman, and P. H. Stone. Relation of vessel wall shear stress to atherosclerosis progression in human coronary arteries. Arterioscler. Thromb. 13:310-315, 1993.

Gidron, Y., H. Gilutz, R. Berger, and M. Huleihel. Molecular and cellular interface between behavior and icute coronary syndromes. Cardiovasc. Res. 56:15-21, 2002.

Guinea, G. V., J. M. Atienza, M. Elices, P. Aragoncillo, ind K. Hayashi. Thermomechanical behavior of human :arotid arteries in the passive state. Am. J. Physiol. Heart Circ. Physiol. 288:H2940-H2945, 2005.

Gurfinkel, E. P., R. L. de la Fuente, O. Mendiz, and B. Mautner. Flu vaccination in acute coronary syndromes 
and planned percutaneous coronary interventions (fluvacs). Eur. Heart J. 25:25-31, 2004.

Hayashi, K., K. Ide, and T. Matsumoto. Aortic walls in atherosclerotic rabbits - mechanical study. /. Biomech. Eng. 116:284-293, 1994.

Hayashi, K., and Y. Imai. Tensile property of atheromatous plaque and an analysis of stress in atherosclerotic wall. I. Biomech. 30:573-579, 1997.

Kelly, A., and W. R. Tyson. Tensile properties of fibre-reinforced metals - copper/tungsten and copper/ molybdenum. /. Mech. Phys. Solids 13:329-350, 1965.

Lavallee, P., V. Perchaud, M. Gautier-Bertrand, D. Grabli, and P. Amarenco.. Association between influenza vaccination and reduced risk of brain infarction. Stroke 33:513518, 2002.

Lin, S. G., X. Y. Yu, Y. X. Chen, X. R. Huang, C. Metz, R. Bucala, C. P. Lau, and H. Y. Lan. De novo expression of macrophage migration inhibitory factor in atherogenesis in rabbits. Circ. Res. 87:1202-1208, 2000.

Madjid, M., M. Naghavi, B. A. Malik, S. Litovsky, J. T. WiUerson, and W. Casscells. Thermal detection of vulnerable plaque. Am. J. Cardiol. 90:36L-39L, 2002.

Maseri, A., and V. Fuster. Is there a vulnerable plaque? Circulation 107:2068-2071, 2003.

Meyers, D. G. Myocardial infarction, stroke, and sudden cardiac death may be prevented by influenza vaccination. Curr. Atheroscler. Rep. 5:146-149, 2003.

Naghavi, M., P. Libby, E. Falk, S. W. Casscells, S. Litovsky, J. Rumberger, J. J. Badimon, C. Stefanadis, P. Moreno, G. Pasterkamp, Z. Fayad, P. H. Stone, S. Waxman, P. Raggi, M. Madjid, A. Zarrabi, A. Burke, C. Yuan, P. J. Fitzgerald, D. S. Siscovick, C. L. de Korte, M. Aikawa, K. E. J. Airaksinen, G. Assmann, C. R. Becker, J. H. Chesebro, A. Farb, Z. S. Galis, C. Jackson, I. K. Jang, W. Koenig, R. A. Lodder, K. March, J. Demirovic, M. Navab, S. G. Priori, M. D. Rekhter, R. Bahr, S. M. Grundy, R. Mehran, A. Colombo, E. Boerwinkle, C Ballantyne, W. Insull, R. S. Schwartz, R. Vogel, P. W. Serruys, G. K. Hansson, D. P. Faxon, S. Kaul, H. Drexler, P. Greenland, J. E. Muller, R. Virmani, P. M. Ridker, D. P. Zipes, P. K. Shah, and J. T. WiUerson. From vulnerable plaque to vulnerable patient - a call for new definitions and risk assessment strategies: Part I. Circulation 108:1664-1672, 2003.

Naghavi, M., P. Libby, E. Falk, S. W. Casscells, S. Litovsky, J. Rumberger, J. J. Badimon, C. Stefanadis,
P. Moreno, G. Pasterkamp, Z. Fayad, P. H. Stone, S. Waxman, P. Raggi, M. Madjid, A. Zarrabi, A. Burke, C. Yuan, P. J. Fitzgerald, D. S. Siscovick, C. L. de Korte, M. Aikawa, K. E. J. Airaksinen, G. Assmann, C. R. Becker, J. H. Chesebro, A. Farb, Z. S. Galis, C Jackson, I. K. Jang, W. Koenig, R. A. Lodder, K. March, J. Demirovic, M. Navab, S. G. Priori, M. D. Rekhter, R. Bahr, S. M. Grundy, R. Mehran, A. Colombo, E. Boerwinkle, C. Ballantyne, W. Insull, R. S. Schwartz, R. Vogel, P. W. Serruys, G. K. Hansson, D. P. Faxon, S. Kaul, H. Drexler, P. Greenland, J. E. Muller, R. Virmani, P. M. Ridker, D. P. Zipes, P. K. Shah, and J. T. WiUerson. From vulnerable plaque to vulnerable patient - a call for new definitions and risk assessment strategies: Part II. Circulation 108:1772-1778, 2003.

Naghavi, M., P. Wyde, S. Litovsky, M. Madjid, A. Akhtar, S. Naguib, M. S. Siadaty, S. Sanati, and W. Casscells. Influenza infection exerts prominent inflammatory and thrombotic effects on the atherosclerotic plaques of apolipoprotein e-deficient mice. Circulation 107:762-768, 2003. Rekhter, M. D., G. W. Hicks, D. W. Brammer, C. W. Work, J. S. Kim, D. Gordon, J. A. Keiser, and M. J. Ryan. Animal model that mimics atherosclerotic plaque rupture. Circ. Res. 83:705-713, 1998.

Sanmartin, M., J. Goicolea, C. Garcia, J. Garcia, A. Crespo, J. Rodriguez, and J. M. Goicolea. Influence of shear stress on in-stent restenosis: in vivo study using $3 \mathrm{~d}$ reconstruction and computational fluid dynamics. Rev. Esp. Cardiol. 59:20-27, 2006.

Stefanadis, C, K. Toutouzas, E. Tsiamis, C. Stratos, M. Vavuranakis, I. Kallikazaros, D. Panagiotakos, and P. Toutouzas. Increased local temperature in human coronary atherosclerotic plaques: an independent predictor of clinical outcome in patients undergoing a percutaneous coronary intervention. /. Am. Coll. Cardiol. 37:1277-1283, 2001.

Timoshenko, S. Analysis of bi-metal thermostats. /. Opt. Soc. Am. 11:233-255, 1925.

Versluis, A., A. J. Bank, and W. H. Douglas. Fatigue and plaque rupture in myocardial infarction. /. Biomech. 39:339-347, 2006.

Wang, K. P., Y. Y. Huang, A. Chandra, and K. X. Hu. Interfacial shear stress, peeling stress, and die cracking stress in trilayer electronic assemblies. IEEE Trans. Components Pack. Technol. 23:309-316, 2000. 